Ann. A beille, I966, $9(4), 323-350$.

\title{
LES MODALITÉS DE L'ADAPTATION DES ABEILLES (APIS MELLIFICA L.) AU MILIEU NATUREL
}

\author{
J. LOUVEAUX
}

Station de Recherches sur l'Abeille et les Insectes sociaux, 91 -Bures-sur-Yvette avec la collaboration technique de :

M. AlBisetti

Laboratoire de Pathologie apicole du Sud-Ouest, 40 -Sabres

M. Delangue

Station expérimentale d'Apiculture, Centre de Recherches agronomiques du Sud-Fst 84- Montfavet

M. TheurkalfF

Station de Recherches sur l'Abeille et les Insectes sociaux, 91 -Bures-sur-Yvette

\section{SOMMAIRE}

L'auteur après avoir décrit les conditions bioclimatiques propres à trois milieux différents (Région Parisienne, Grande I,ande de Gascogne, environs d'Avignon) expose les résultats d'expériences de permutation de colonies d'abeilles de la même race géngraphique (Apis mellifica mellifica) mais originaires de deux des milieux décrits. Après leur déplacement les colonies conservent des traits de comportement adaptés au milieu antérieur. L'auteur discute de l'origine et de l'importance du phénomène en matière de sélection des abeilles.

\section{INTRODUCTION}

Aucune espèce animale ou végétale ne peut se maintenir dans un milieu donné si elle ne présente pas les caractères adaptatifs, anatomiques et physiologiques, nécessaires à la vie au sein de ce milieu. Ces caractères sont susceptibles d'être larges, étroits ou très étroits, ce qui influe, bien entendu, sur la répartition géographique des espèces.

Les caractères adaptatifs ne sont pas toujours fixés d'une façon rigide. Certains organismes sont susceptibles de supporter d'importantes variations dans leurs conditions de vie; leur adaptation à un nouveau milieu se traduit par des modifications morphologiques ou physiologiques auxquelles on donne généralement le nom d'acco- 
modats (CUÉNOT et T'élRY, I95r). Ces modifications ne s'inscrivent pas dans le patrimoine héréditaire puisque le retour aux conditions normales entraîne, en principe, leur disparition. Il existe toutefois au sein des espèces des races physiologiques dont les caractères sont stables. Ces phénomènes sont suffisamment connus pour que nous examinions directement le cas particulier que constitue l'Abeille (Apis mellifica L.).

Par son anatomie 1'Abeille est adaptée à la récolte du pollen et du nectar. Si l'on considère la flore qu'elle utilise de façon courante on constate que son adaptation anatomique au monđe végétal est très large. L'Abeille n'est pas inféodée à une espèce ni même à un groupe botanique limité. Bien au contraire, ses organes de récolte lui permettent de visiter avec profit les plantes les plus diverses. On notera en particulier qu'elle peut utiliser aussi bien des plantes à chatons (Corylus) que des Ombellifères ou des Papilionacées. Seules les fleurs à corolle trop profonde s'opposent. à ses visites.

I'autre part, on constate que l'Abeille supporte bien les climats les plus variés. On peut la qualifier de cosmopolite puisqu'on la trouve presque jusqu'au cercle polaire et jusqu'à l'équateur. Elle a rapidement conquis le Nouveau Monde et l'Australie sans que 1'homme ait été obligé de la protéger de façon particulière. Son absence de certaines régions équatoriales n'est pas liée au climat mais plutôt, semble-t-il, à 1'abondance locale des ennemis naturels.

Sur le plan nutritionnel l'Abeille est très plastique. Non seulement elle utilise le nectar et le pollen d'un grand nombre d'espèces végétales mais elle peut récolter et utiliser pour son alimentation des produits de remplacement tels que le saccharose pur, diverses farines, des levures séchées, etc.

Son cycle annuel est susceptible de variations importantes. Alors que sous les tropiques les conditions climatiques lui permettent une activité à peu près continue, elle ne bénéficie au Canada, en Sibérie ou en Norvège que de quelques mois de beau temps chaque année et doit faire face à un hivernage long et dur.

L'Abeille se présente donc au premier abord comme un Insecte essentiellement cosmopolite et plastique. En réalité il faut tenir compte du fait que l'espèce Apis mellifica se subdivise en un certain nombre de races géographiques, lesquelles comprennent à leur tour des formes locales sans doute fort nombreuses mais qui, du point de vue systématique, sont pratiquement insaisissables même avec l'aide de la biométrie (DROEGE, I960).

Les races géographiques, qui sont en nombre limité, constituent des unités systématiques relativement bien définies et génétiquement stables. Elles présentent des caractères morphologiques et physiologiques distincts ; d'autre part leur comportement présente, lui aussi, des particularités très nettes. Ces caractères peuvent être considérés comme adaptés au milieu d'origine. Ce sont donc, en fait, les races géographiques de 1'Abeille qui, par leurs adaptations particulières ont permis à l'espèce de s'étendre sur la plus grande partie des continents. I1 est certain que l'adaptation est assez poussée pour qu'il soit pratiquement impossible de faire vivre certaines abeilles africaines dans un pays nordique. Cependant, les races géographiques des latitudes moyennes peuvent s'accommoder souvent de climats extrêmes, ce qui prouve que l'adaptation au milieu chez les différentes races géographiques n'est jamais devenue rigide et laisse place à de larges possibilités d'acclimatation. Les observations de LUNDER (I953) confirment cette idée. Dans les conditions de la 
Norvège on a pu maintenir pendant plusieurs décennies à l'état pratiquement pur un certain nombre de colonies d'abeilles appartenant à des races géographiques diverses (A.m. ligustica, A. m. carnica, A.m. caucasica). Les colonies transplantées ont surmonté les difficultés provenant du changement de climat mais elles ont conservé leurs traits de caractères biologiques et physiologiques essentiels. En particulier, leur cycle annuel, adapté nécessairement aux conditions locales, a conservé cependant les tendances caractéristiques de la race (position du maximum de développement à l'intérieur du cycle; importance relative des populations d'été et d'hiver).

Nous sommes donc à l'heure actuelle relativement bien renseignés sur les caractères essentiels des principales races géographiques de l'Abeille. Nous savons que ces caractères sont mesurables et que, sauf hybridation, ils semblent bien se conserver pour l'essentiel lorsqu'on procède à une tentative d'acclimatation. Il se produit une adaptation généralement suffisante pour permettre la survie mais, à notre échelle des temps, la race introduite ne devient pas la phénocopie de la race locale dont l'adaptation est millénaire.

Par contre, lorsqu'on abandonne cette unité systématique très réelle qu'est la race géographique pour explorer le domaine des formes locales de l'Abeille que le systématicien ne peut plus saisir, on s'aperçoit qu'on manque de données objectives. On sait, parce que les apiculteurs l'ont maintes fois observé, que les abeilles d'une race géographique donnée n'ont pas tontes le même comportement, ce qui se traduit sur le plan pratique par des différences sensibles de rendement. Empiriquement, les apiculteurs professionnels savent dans quelle région ils doivent de préférence faire leurs achats d'abeilles pour obtenir de bons résultats. Ėn fait, il reste à rechercher si ces différences de comportement sont réelles et, dans 1'affirmative, comment elles se manifestent. Iin supposant que la notion de race locale doive être admise dans son sens le plus étroit, il paraît intéressant de définir les processus intimes de l'adaptation au milieu ambiant. Encore faut-il préalablement définir ce milieu.

Le présent travail, qui porte maintenant sur quatre années et qui se poursuit, a pour objet l'étude de quelques cas précis d'adaptation de l'abeille noire commune (A. m. mellifica) à différents milieux choisis pour leurs caractères très particuliers.

\section{MATÉRIEL, ET MÉTHODES}

L'étude des modalités de l'adaptation des abeilles au milieu naturel suppose : d'une part, des méthodes d'observation applicables aux différents milieux choisis pour conduire les expériences, d'autre part, des méthodes permettant l'observation des réactions des colonies d'abeilles face à ce milieu.

Io Lieux des expériences

Les expériences ont eu lieu :

— à Bures-sur-Xvette (Essonne), à $25 \mathrm{~km}$ au sud-ouest de Paris ;

- à Sabres (Landes), localité située sensiblement à mi-chemin de Bordeaux et de la frontière espagnole ;

- à Montfavet (Vaucluse) à $7 \mathrm{~km}$ à l'est d'Avignon. 


\section{$2^{0}$ Méthodes d'étude du milieu naturel}

Facteurs climatiques. - Étant donné le caractère de ce travail nous n'avons pas pris en considération le micro-climat des ruchers expérimentaux. Ce facteur est certes plein d'intérêt mais il nous a paru plus important de ne tenir compte que des caractéristiques générales du climat régional. Grâce aux relevés de la Météorologie nationale $\left(^{1}\right)$ relatifs aux stations les plus rapprochées des lieux de travail nous obtenons un profil annuel moyen beaucoup plus significatif qu'un nombre forcément très limité d'observations locales. Nous n'avons pris en considération que la température moyenne et la pluviométrie.

Flore. - La flore de chacun des lieux servant aux expériences est connue par étude directe sur le terrain dans un périmètre de 2 à $3 \mathrm{~km}$ autour de chaque rucher. Elle est connue également de façon indirecte par l'examen qualitatif des récoltes de pollen obtenues au moyen des trappes classiques.

Autres facteurs. -- L'altitude des trois lieux d'expérience est inférieure à $\mathrm{I} 00 \mathrm{~m}$; ce facteur peut donc être éliminé. I.e rôle de la nature du sol est fondamental mais il n'est étudié qu'à travers son influence sur la flore.

\section{$3^{\circ}$ Les colonies d'abeilles}

Les colonies d'abeilles utilisées appartiemnent toutes à la race noire commune (A. m. mellifica). Les caractères biométriques utilisés pour les contrôles sont los caractères classiques tels qu'on les trouve décrits dans les ouvrages de base (Goetze 1964, RlitTner 1963). Toutes les colonies présentant un signe d'hybridation avec une autre race géographique ont été éliminées.

Les colonies dites " de Bures-sur-Yvette " appartiennent au rucher de la Station de Recherches sur l'Abeille et les Insectes Sociaux et peuvent être considérées comme représentatives de l'abeille locale du Bassin parisien.

les colonies dites "de Sabres "appartiennent au rucher du Laboratoire de Pathologie apicole du sud-Ouest. Elles proviennent toutes de ruchers landais sédentaires soigneusement choisis pour leur isolement. Rappelons que Louss ( 1963 ) étudiant l'abeille landaise du point de vue biométrique ne l'a pas trouvée significativement différente de l'abeille noire d'autres régions de France.

l.es colonies dites " de Montfavet "appartiennent aux souches de la race noire commune sélectionnées depuis $195^{6}$ par la Station expérimentale d'Apiculture et dont l'étude biométrique a été faite récemment par I'RESNAYE (1965).

Pour chaque série d'expériences les reines sont de même âge. Ellles sont marquées et clippées. On ne tient compte que des résultats obtenus avec des colonies n'ayant pas essaimé et n'ayant pas changé de reine à la suite d'un orphelinage.

\section{$4^{\circ}$ Méthodes d'observation des colonies d'abeilles}

l)ans chacun des ruchers expérimentaux on procède aux mêmes dates très exactement à un certain nombre d'observations:

Poids des ruches - Les observations sont hebdomadaires. On utilise le pèse-ruches mis au point pour la pesée rapide par Fresnaye (196r). Les pesées sont faites avec des instruments semblables et en observant les mêmes règles en ce qui concerne la déduction des poids morts.

Récolte du pollen. - Les relevés sont hebdomadaires. On utilise la trappe à pollen en position supérieure (LAVIE et FRESNAYE, 1963) à trois rangées de trous. La libération des mâles se fait aux mêmes dates dans les trois ruchers par ouverture d'un orifice spécial à l'arrière de la trappe. Cet orifice est muni d'un chasse-abeilles qui interdit la rentrée des butineuses. La récolte est séchée à l'étuve à $40^{\circ}$ pendant deux jours et elle est ensuite pesée. Un échantillon homogène de ro $\mathrm{g}$ est prélevé et sert à faire une préparation microscopique pour l'identification des sources de pollen.

Surfaces de couvain. - Aux dates fixées en début de campagne les surfaces de couvain de charque colonie en expérience sont mesurées selon la méthode de FrEsNAYE (I962).

Autres observations. - Toutes les colonies en expérience font l'objet d'une surveillance continuelle en ce qui concerne la présence de la reine, l'essaimage et l'état sanitaire.

D'une façon générale, toutes les précautions sont prises pour rendre les observations aussi comparables que possible Tout le matériel est du même modèle (ruches Dadant io cadres) et peint au moyen de la même peinture à pigment aluminiun. Dans chaque rucher toutes les ruches ont la même orientation mais les lots sont mélangés de façon à éviter un effet de position. L'espace-

(1) Direction de la Mlétéorologie nationale. Résumé mensuel (lu 'lemplss en France. 
ment des ruches et l'utilisation de panneaux colorés devant le trou de vol permettent d'éviter la dérive (FRESNAYE I963). Tous les nourrissements sont comptabilisés et réduits au strict minimun; ils ne sont jamais distribués d'une façon qui pourrait conduire à une stimulation artificielle importante de la ponte de la reine.

\section{$5^{\circ}$ Schéma général des expériences}

On distingue d'une part les expériences ayant pour objet de définir des caractéristiques générales du cycle biologique des abeilles en un lieu donné. Dans ce cas un petit lot de ruches, généralement trois, est mis en observation selon la méthode qui vient d'être exposée. On ne procède à aucune opération susceptible d'avoir une influence sur le développement de la colonie (déplacement de cadres, introduction de bâtisses vides) ; on se contente de poser les hausses lorsque cette opération est normalement nécessaire. Les données chiffrées recueillies sont utilisées pour déterminer le cycle biologique des colonies.

D'autre part, on procède à des expériences de permutation qui consistent à échanger un nombre égal de colonies comparables entre deux ruchers expérimentaux. La comparaison des résultats obtenus dans les lots ainsi constitués renseigne sur le degré d'adaptation des abeilles au milieu nouveau.

\section{RÉSULTATS}

\section{I. - LES CARACTÉRISTIQUES DES MILIEUX ÉTUDIÉS}

L'étude de 1'adaptation des colonies d'abeilles à un milieu donné suppose une connaissance préalable de ce milieu. Les observations dont nous disposons maintenant nous permettent de connaître pour chacun des lieux étudiés l'essentiel des facteurs climatiques ainsi que le rythme des principales floraisons. Nous examinerons successivement les trois milieux ayant fait jusqu'ici l'objet d'observations.

\section{Bures-sur-Yvette}

Cette localité est située dans la vallée de Chevreuse. Le poste d'observations météorologiques le plus proche et pour lequel on dispose de moyennes trentenaires est celui de Paris-Orly où la pluviométrie est de l'ordre de $620 \mathrm{~mm}$ par an avec

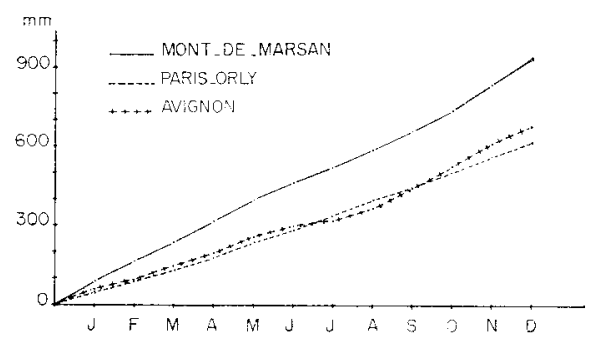

FIG. I. - Pluviométrie comparée à Mont-de-Marsan, Paris-Orly et Avignon

Courbes cumulatives. Moyennes trentenaires établies par la Météorologie nationale

une répartition à peu près homogène tout au long de l'année (fig. I). La courbe des températures moyennes mensuelles (fig. 2) a son maximum en juillet $\left(\mathrm{I} 9^{\circ}\right)$. Il faut attendre avril pour que la température moyenne dépasse $\mathrm{I}^{\circ}$; la moyenne d'octobre n'est déjà plus que de $\mathrm{II}^{\circ} 4$. Décembre et janvier sont relativement froids avec $3^{\circ} 4$. 
La courbe du poids de pollenr écolté à la trappe (fig. 3) présente une allure générale caractéristique que nous retrouvons toujours depuis de nombreuses années (LouveAuX, I958) avec les inévitables variations d'amplitude qui tiennent au caractère particulier de chaque saison. On distingue cinq périodes principales caractérisées par la dominance d'une plante ou d'un groupe de plantes. La figure 3 donne le détail de ces périodes. On remarquera surtout que $90 \mathrm{p}$. Ioo du pollen se trouve récolté avant le $I_{5}$ juillet et $75 \mathrm{p}$. Ioo entre le début de mai et le I $_{5}$ juillet, c'est-à-dire sur moins de 80 jours. Si l'on examine les courbes de poids des ruches (fig. 7) on retrouve le même phénomène : aucun gain de poids avant la mi-avril, perte de poids constante à partir du $\mathrm{I}_{5}$ juillet. Toute la récolte se fait donc sur trois mois. La courbe des surfaces de couvain (fig. 9) montre un développement rapide des colonies en avril et un palier en mai et juin ; à la fin du mois de juillet le niveau de l'élevage est déjà très bas et le mois de septembre voit l'arrêt à peu près complet de la ponte.

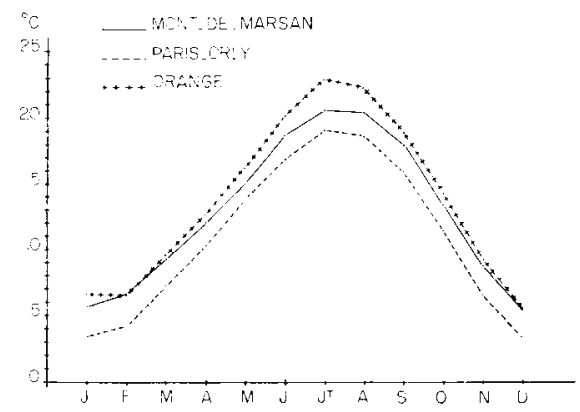

Iira. 2. - Températures moyennes à Mon-de-Marsan, Paris-Only el Orange.

Moyennes trentenaires établies par la Météorolorric nationale.

Vingt années d'observations à Bures-sur-Yvette nous démontrent de façon claire que le cycle biologique des abeilles y est essentiellement printanier et à un seul sommet : toute 1'activité des abeilles est concentrée sur trois mois ( 15 avril-I5 juillet). Nous savons d'autre part par expérience que pour obtenir les abeilles nécessaires aux expériences tout au long de 1'année nous avons toujours été obligés de recourir à des procédés de forçage.

\section{Sabres}

Le rucher du Laboratoire de Pathologie apicole du Sud-Ouest est situé en plein centre de la Grande-Lande. Nous avons déjà étudié les caractères essentiels de ce milieu naturel (Louveaux et AlbisetTi, I963). Couturier (I950) l'avait d'ailleurs précédemment décrit dans un article sur l'apiculture landaise. Le poste d'observations météorologiques le plus proche est celui de Mont-de-Marsan (fig. I et 2). La pluviométrie annuelle y est de $940 \mathrm{~mm}$ avec une répartition presque uniforme sur 1'ensemble de l'année, l'été étant à peine moins pluvieux que les autres saisons. La courbe des moyennes mensuelles de températures est presque parallèle à celle de ParisOrly avec un décalage de $2^{\circ}$ environ. Les températures supérieures à $10^{\circ}$ vont du début de mars à la mi-octobre. La période utile de butinage est donc assez longue. Il convient toutefois de noter que les écarts de température au cours de la journée 
sont très importants; nous avons pu nous en rendre compte en examinant de façon plus détaillée les relevés de la Météorologie nationale.

Ce facteur peut avoir une influence assez néfaste sur le développement des colonies au printemps.

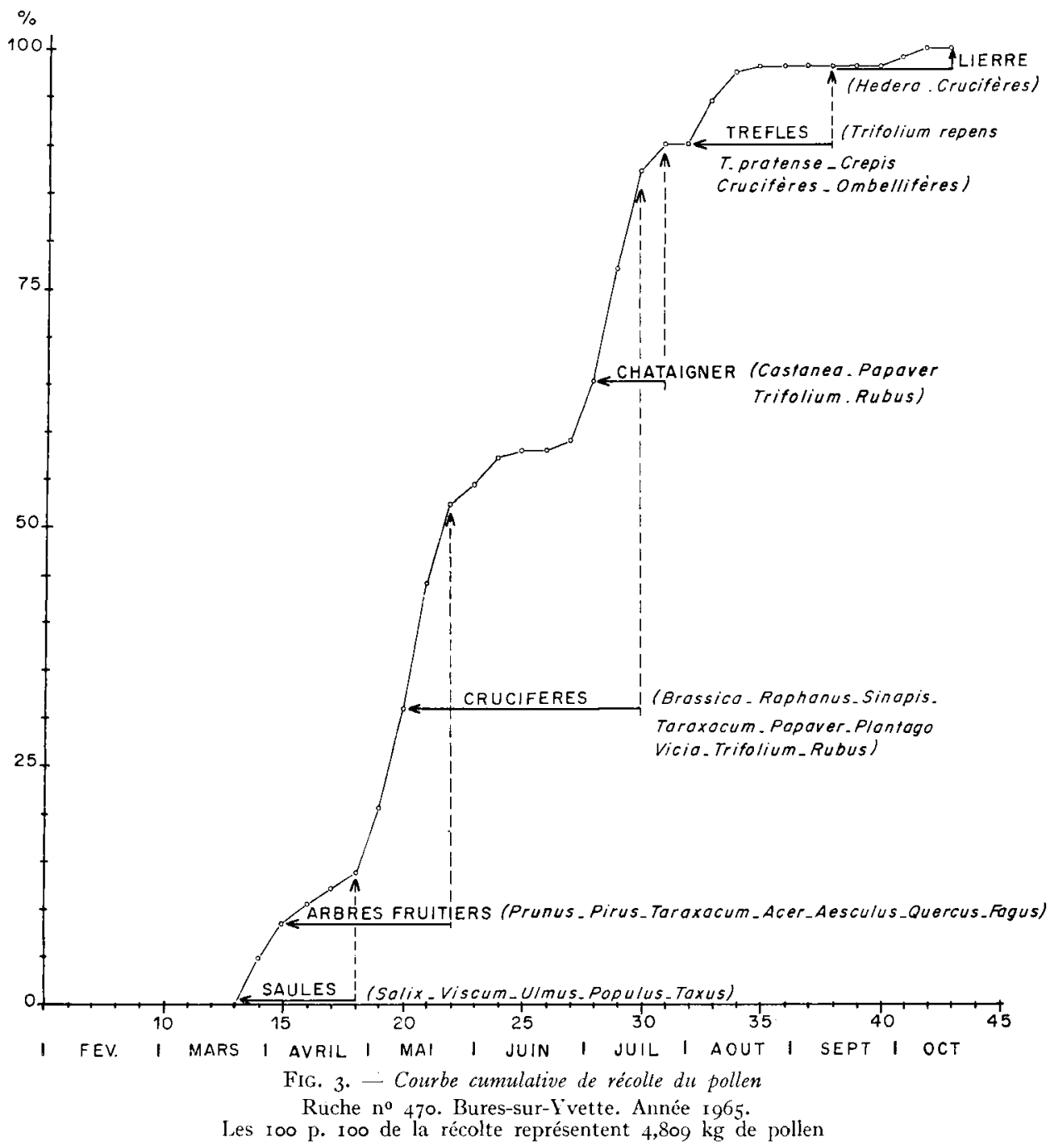

La courbe de récolte du pollen (fig. 4) montre l'existence de quatre périodes distinctes. On notera surtout l'absence de pollen des arbres fruitiers et le rôle très secondaire joué par les Papilionacées, à part Ulex. Au I5 juillet il n'y a encore que $50 \mathrm{p}$. Ioo du pollen total qui soit récolté. Au I5 août il reste encore $25 \mathrm{p}$. roo du pollen à rentrer et ce pollen provient surtout de la Callune. Les courbes de poids des ruches obtenues sur trois années successives montrent que les gains de poids printaniers sont faibles (fig. 6). La miellée la plus forte, celle de Callune, intervient 
à partir de la fin du mois d'août et se prolonge jusqu'en octobre. Nos courbes coïncident de façon très satisfaisante avec celles de CouTURIER (I950) concernant la période I937-I942, ce qui montre la stabilité du milieu landais sur près de 30 ans. La courbe des surfaces de couvain (fig. 9) présente une ascension lente au printemps ; l'élevage se poursuit activement jusqu'en octobre.

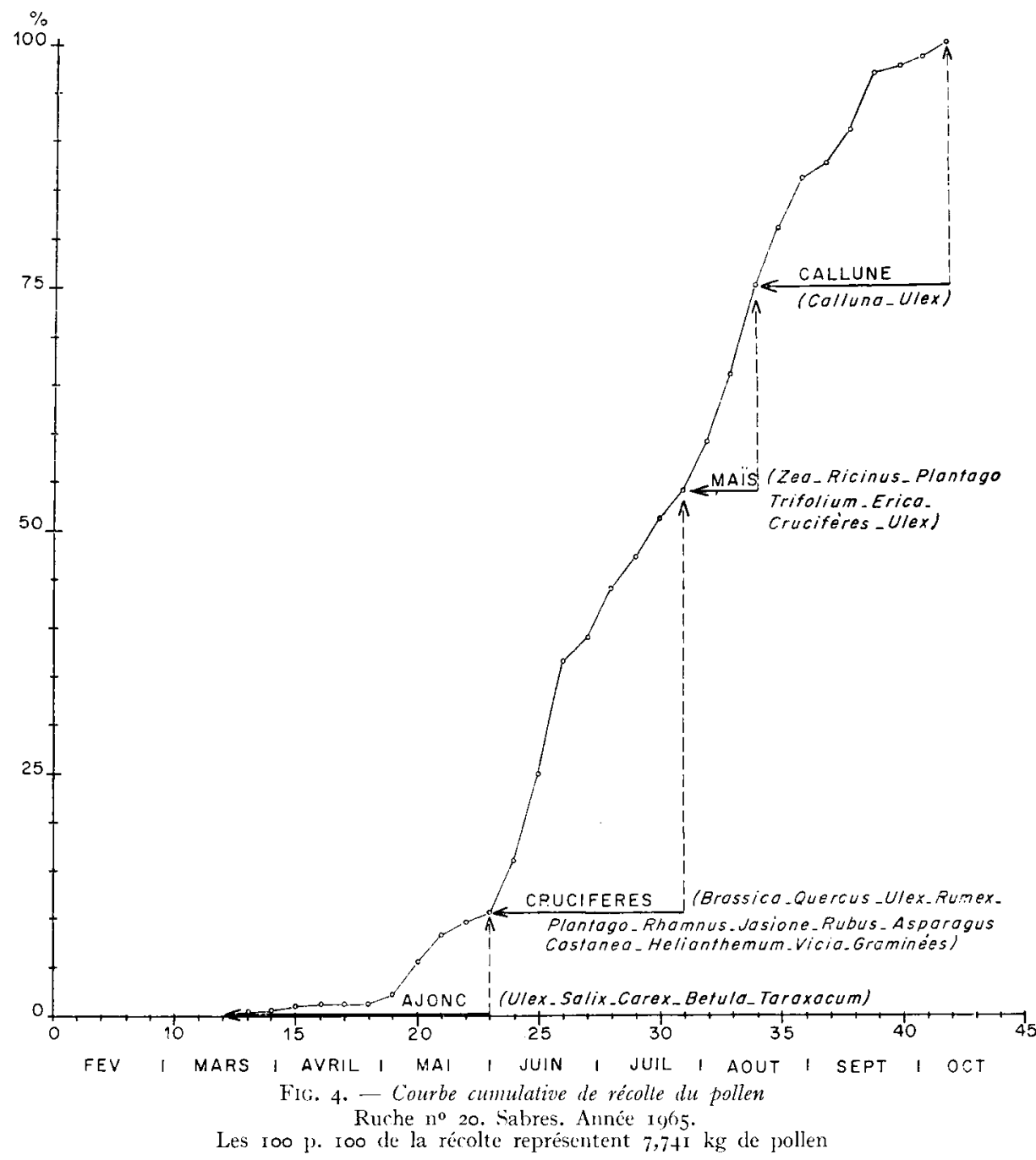

Nous sommes donc à Sabres dans une région de climat humide et assez chaud où la flore de printemps est peu abondante. Iille ne permet pas un développement rapide des colonies; l'activité maximum est estivale et même automnale.

\section{Montfavet}

Le rucher d'observations de la Station expérimentale d'Apiculture se trouve à quelques centaines de mètres de la Durance, dans une zone continuellement irriguée 
où dominent les cultures maraîchères, fruitières et fourragères. La pluviométrie moyenne d'Avignon, station météorologique la plus proche, est de $680 \mathrm{~mm}$ par an. La pluviométrie estivale est faible. Ne disposant pas de relevés trentenaires de température pour Avignon mème, nous avons pris en considération les moyennes annuelles d'Orange. L'été est chaud ( $23^{\circ}$ en juillet) mais l'hiver n'est pas plus doux qu'à Montde-Marsan. La période d'activité possible des abeilles va du début de mars à la mioctobre.

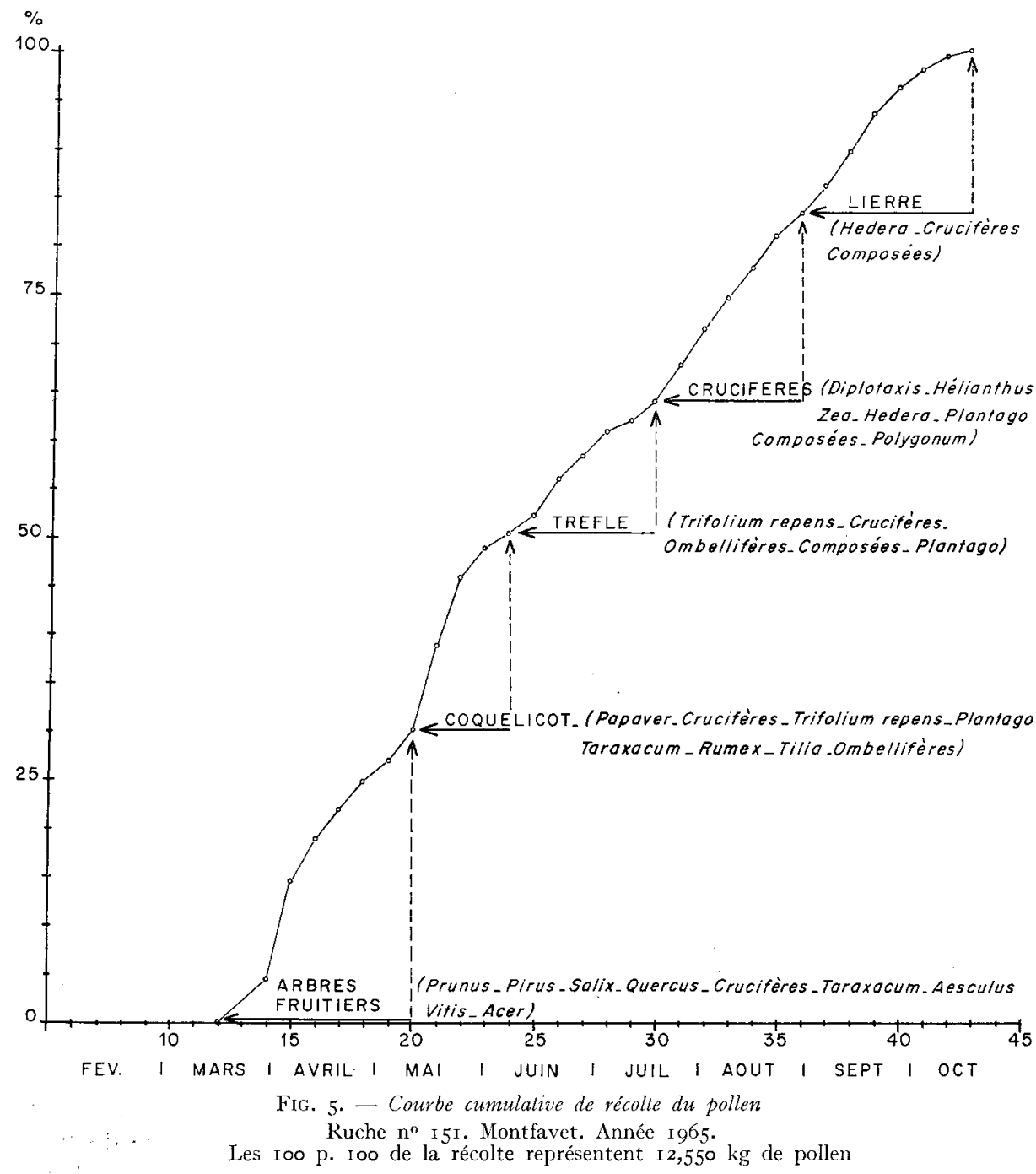

La courbe de récolte du pollen (fig. 5) se rapproche d'une droite allant régulièrement du I5 mars au I5 octobre sans présenter de très grosses fluctuations. On peut distinguer 5 périodes principales. On constate par ailleurs que les arbres fruitiers et les Papilionacées jouent un rôle essentiel). La courbe de variation de poids des 
ruches (fig. 8) montre un maximum au début d'avril au moment de la floraison des arbres fruitiers. Une période relativement creuse succède à ce maximum; il faut attendre la floraison des trèfles pour que la courbe de poids reprenne sa forme ascendante. Elle la conserve jusqu'en octobre, ce qui s'explique par le fait qu'on se trouve en zone irriguée et que la sécheresse estivale est sans effet. La courbe des surfaces de couvain met en relief le maximum printanier. Pendant tout l'été l'élevage se maintient à un bon niveau bien que la courbe soit descendante de façon continue.

La région de Montfavet peut être considérée comme printanière précoce et secondairement estivale du fait de l'irrigation. L'activité des abeilles est continue de mars à octobre. La flore est abondante et variée mais la note dominante est donnée par les arbres fruitiers au printemps et par le Trèfle blanc en été.

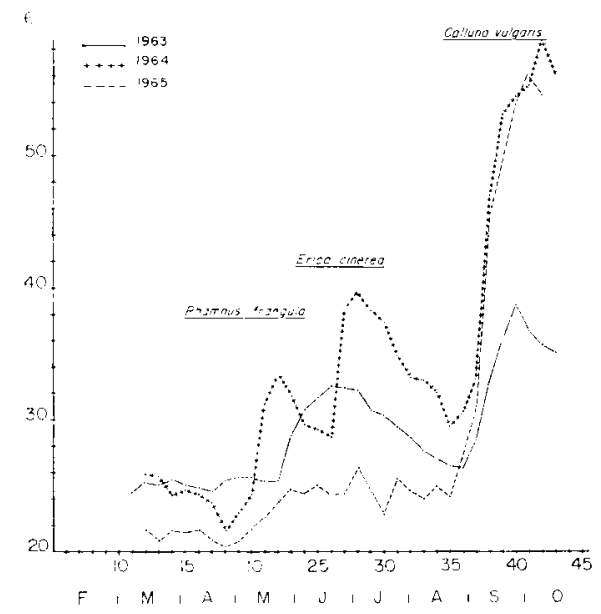

Fig. 6. - Courbes de poids des ruches à Sabres pendanl les années 1963, 1964 et 1965.

Ig63. Ruche sur bascule. Abeilles landaises.

I 964 . Moyenne des poids de 5 ruches. Abeilles landaises.

1965. Variations de poids de la ruche $n^{\circ}$ 17. $\Lambda$ beilles landaises.

\section{Conclusion}

Il résulte de l'étude de ces trois types de milieux qu'à l'intérieur d'un pays comme la France 1'Abeille peut se trouver soumise à des conditions de climat et de flore présentant une grande diversité. Si nous avions pu faire l'étude d'autres biotopes, il est probable que nous aurions mis en évidence l'existence d'autres régimes, notamment en fonction de l'altitude et de la flore des hautes montagnes. En résumé, on peut déjà opposer Bures-sur-Yvette et Montfavet à Sabres. Ces deux premières localités sont du régime printanier en ce sens que les floraisons les plus importantes se situent en début de saison ; les arbres fruitiers jouent un rôle essentiel ; ils conditionnent le développement précoce des colonies. Montfavet se distingue de Buressur-Yvette par le fait que grâce à l'irrigation la période estivale reste productive alors qu'à Bures-sur-Yvette, qui est la plus sèche des trois localités étudiées, il n'y a jamais récolte estivale.

Sabres est d'un régime tout différent qu'on peut qualifier d'estivo-automnal. Le printemps, par suite de la déficience de la flore et peut-être aussi par suite des écarts 
de température dont il a été question plus haut, n'est pas une période de développement rapide des colonies. L'essentiel de l'activité se manifeste en été et au début de l'automne (fig. 8, 9 et Io).

Nous noterons ici que nous rejoignons les observations de KocH (I962, I964, $\mathrm{I} 965 a, b$ et $c, \mathrm{I} 966)$ sur les régions mellifères de la $\mathrm{R}$. $\mathrm{D}$. A. et sur l'importance des données que l'on peut recueillir au moyen de la pesée régulière des ruches.

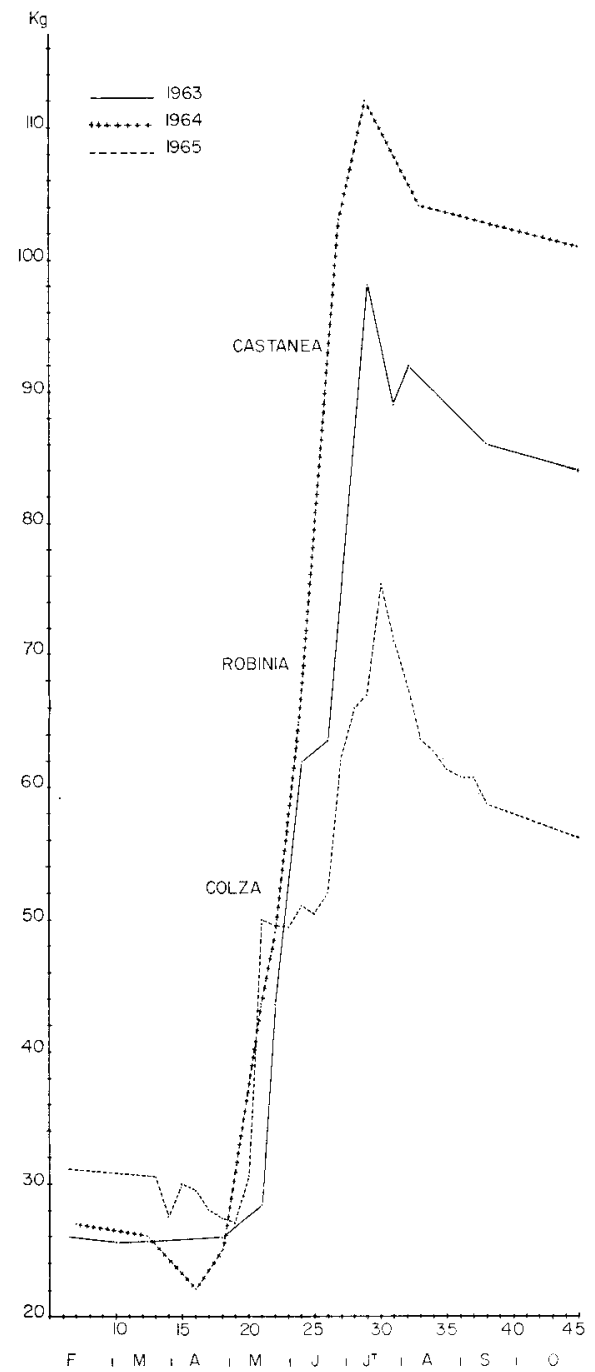

FiG. 7. - Courbes de poids d'une ruche sur bascule sous abri à Bures-sur-Yvelle: - pendant les années rg63-1964. Abeilles de race italienne.

- en 1965 . Variations de poids de la ruche $n^{\circ} 470$. Abeilles de race noire locale.

On peut penser, a priori, que s'il y a adaptation des abeilles à un régime bioclimatique, cette adaptation devrait se manifester par l'existence d'un rythme biologique calqué sur ce régime. Autrement dit, l'abeille landaise serait adaptée à une 


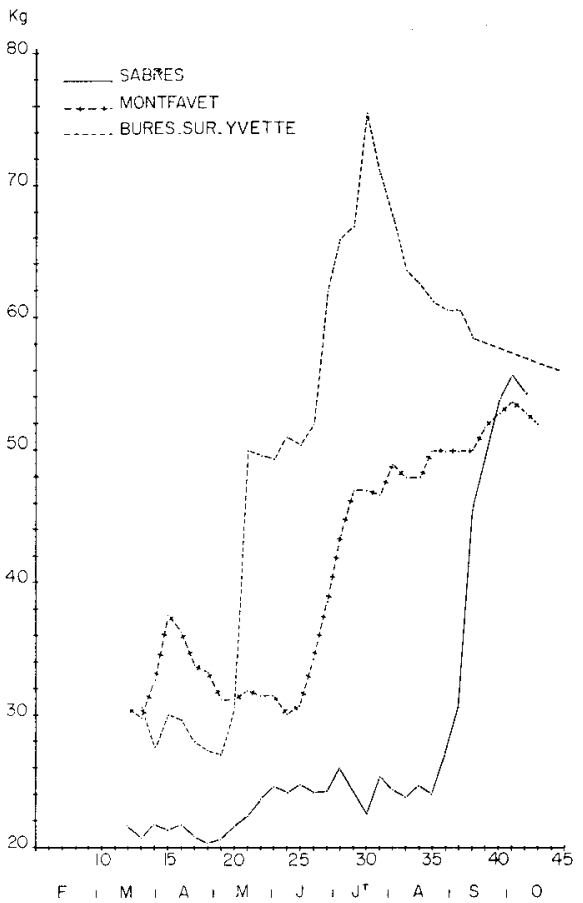

FIG. 8. - Comparaison des courbes de variation de poids en 1960 à Bures-sur-Yvette Ruche $n^{0} 470$ Abeilles de race noire locale à Montfavet Ruche $n^{\circ}$ I 5 I Abeilles de race noire locale à Sabres Ruche no 17 Abeilles de race noire locale

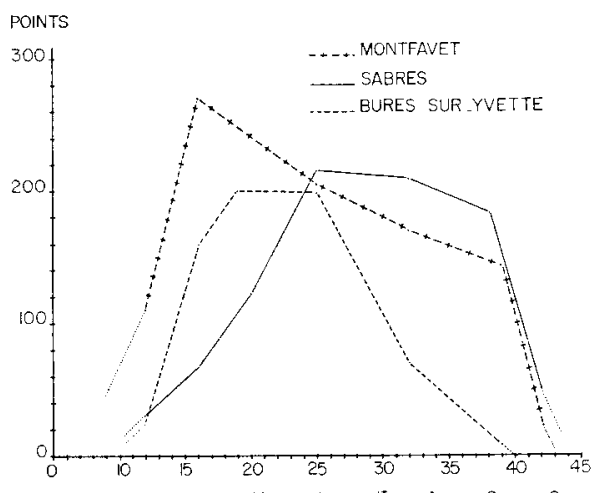

FIG. 9. - Courbes de surface du couvain en 1965

à Bures-sur-Yvette Ruche $n^{\circ} 470$ Abeilles de race noire locale à Montfavet Ruche no I5I Abeilles de race noire locale à Sabres Ruche $\mathrm{n}^{\circ}$ i 7 Abeilles de race noire locale Les surfaces sont exprimées en points valant $40 \mathrm{~cm}^{2}$. 
flore tardive caractérisée par l'abondance des Éricacées tandis que 1'Abeille de l'llede-France et celle de Provence, le seraient à une flore essentiellement précoce caractérisée par la dominance des Rosacées et des Papilionacées.

Les expériences dont l'exposé va suivre devraient nous éclairer sur la valeur de cette hypothèse.

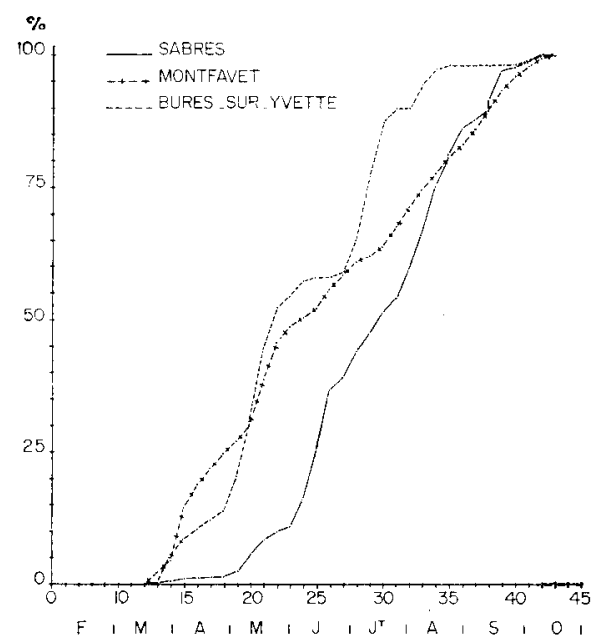

FIG. 10. - Courbes cumulatives de récolle du pollen

Résultats exprimés en p. Ioo du poids total du pollen récolté. Année ro65
à Bures-sur-Yvette
à Montfavet
Ruche $\pi^{\circ} 470$
Abeilles de race noire locale
Ruche $n^{0}$ I $5 \mathrm{I}$ Abeilles de race noire locale
à Sabres
Ruche $n^{\circ} 20$
Abeilles de race noire locale

\section{II. - LES MODALITÉS DE L'ADAPTATION AU MILIEU NATUREL,}

\section{Infuence d'un déplacement simple à courte distance}

Dans un tout premier stade de nos expériences sur l'adaptation des abeilles au milieu naturel nous avons recherché quelle pourrait être l'influence d'un déplacement simple à courte distance sur le comportement des colonies. Nous avons donc comparé des colonies sédentaires n'ayant jamais quitté leur rucher d'origine à des colonies transhumantes amenées d'une région voisine un peu avant le début de la miellée de Callune. L'année I 962 au cours de laquelle ces essais furent faits ayant été climatiquement anormale du fait d'une sécheresse persistante, les résultats obtenus et publiés en I963 (LoUvEAUX et AlBISETTI) ne peuvent pas être considérés comme très significatifs. Les différences de comportement observées tiennent plus aux différences d'état des colonies de chaque lot qu'à une réaction caractéristique à l'égard de la miellée de Callune.

Le même type d'expérience a été repris en I963. Cette fois deux lots de trois colonies ont été comparés. Dans le premier lot il s'agissait de colonies de race landaise locale restant pendant toute l'année dans la Grande-Lande; le second lot était constitué par des colonies de la même race locale mais soumises à transhumance, 
c'est-à-dire qu'elles passèrent la période hivernale Ig62-63 en bordure de la forêt, en Chalosse, ainsi que tout le printemps, et ne furent amenées au rucher de la GrandeLande qu'au début de juillet 1963 . Pendant toute l'année on procéda à des mesures de surface du couvain et à des pesées de la récolte du pollen mais, faute d'équipement, on ne put faire les pesées hebdomadaires des ruches. Une ruche sur bascule fut toutefois installée à Sabres ce qui permit d'obtenir une courbe intéressante.

L'année rg63 fut sensiblement normale du point de vue des conditions météorologiques. Une miellée de Callune eut lieu en août-septembre mais ne fut pas très intense. Aucune différence significative de comportement ne fut notée entre les colonies transhumantes et les colonies sédentaires. Ceci peut tenir au fait que dans les deux lots il s'agissait d'abeilles landaises. Étant donné que les abeilles de Chalosse transhument régulièrement dans 1a Grande-Lande en été on peut supposer qu'elles sont aussi bien adaptées à la miellée de Callune que les abeilles sédentaires.

Un point intéressant fut toutefois noté. Les ruches ayant débuté la saison en Chalosse récoltent nettement plus de pollen au total que les ruches sédentaires. La différence provient des conditions de flore beaucoup plus favorables en Chalosse que dans la lande pendant tout le printemps. Le tableau I permet d'évaluer l'importance des différences constatées.

Parallèlement on note un développement plus précoce des ruches de Chalosse, la différence étant de l'ordre de deux semaines. Notons qu'il ne s'agit que d'un décalage des courbes qui restent par ailleurs très comparables.

Étant donné le caractère plutôt négatif des expériences de 1962 et 1963 en ce qui concerne l'adaptation nous ne nous étendrons pas davantage sur les résultats obtenus au cours de ces deux années et nous verrons plutôt comment fut reprise la question en 1964 .

\section{Résultats d'une expérience de permutation de populations}

N'ayant pas obtenu de résultats significatifs en travaillant avec des colonies soumises à un déplacement simple et à courte distance nous avons organisé en Ig64 une expérience plus importante comportant une permutation de populations. Cinq ruches landaises furent expédiées à Bures-sur-Yvette pendant 1'hiver I963-64 tandis que cinq ruches de Bures-sur-Yvette étaient envoyées à leur place à Sabres. Autant de ruches témoins furent réservées de telle sorte que 20 colonies (Io à Bures-surYvette et to à Sabres) servirent aux essais. Cette fois nous disposions de tout le matériel voulu et les observations purent porter de mars à octobre sur le poids des ruches, le poids et la nature des récoltes de pollen ainsi que sur l'étendue du couvain. Voyons les résultats obtenus (voir également : LouvEaux I965).

- Récoltes de pollen. Jusque vers le $\mathrm{I} 5$ mai les courbes cumulatives du poids de pollen récolté se recouvrent de façon à peu près parfaite aussi bien à Bures-surYvette qu'à Sabres (fig. II et I2). A partir du I5 mai, des différences se manifestent nettement.

A Bures-sur-Yvette les ruches indigènes montrent une intense activité jusque vers la fin de juillet, récoltant ensemble près de $24 \mathrm{~kg}$ de pollen contre $\mathrm{I} 8 \mathrm{~kg}$ pour es ruches landaises. A partir de la fin de juillet les courbes sont parallèles avec une légère tendance pour les ruches landaises à récolter davantage. Qualitativement, 
une différence importante apparaît : toutes les ruches landaises récoltent en masse un pollen de Composée alors que les ruches indigènes travaillent presque exclusivement sur des Crucifères.

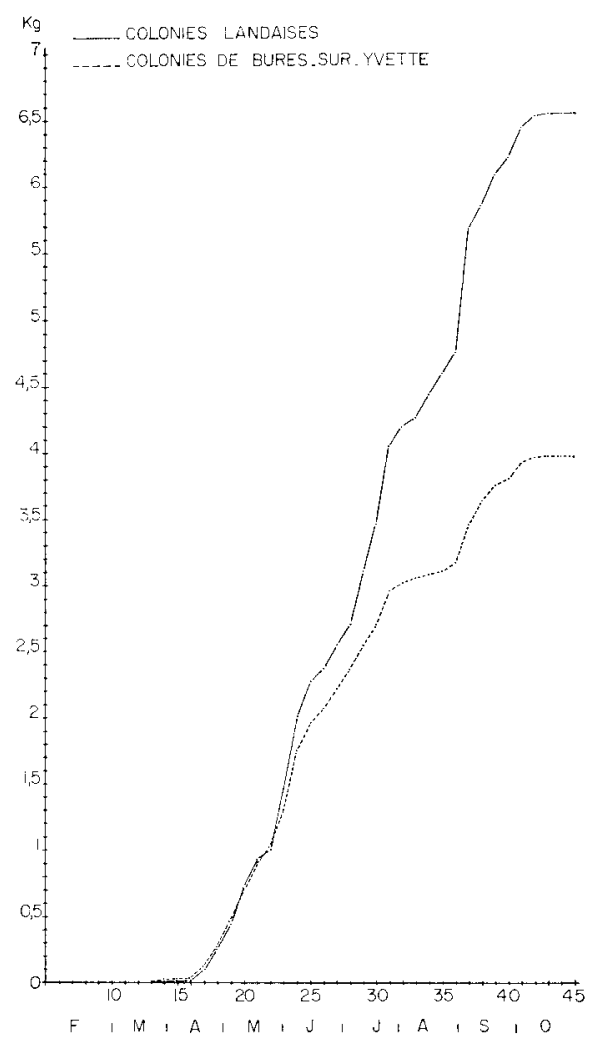

Fig. r. - Courbes cumulatives des récolles de pollen à Sabres.

Année 1964.

D'unc part, moyenne des récoltes des colonies landaises.

D'autre part, moyenne des récoltes des colonies originaires de Bures-sur-Yvette.

Poids exprimés en valeur absolue.

A Sabres, ce sont les ruches landaises qui prennent la tête à partir du I5 mai mais les différences restent faibles jusqu'au début de la floraison des Bruyères. A ce moment les récoltes de pollen des ruches landaises croissent très rapidement alors que celles des ruches importées de Bures-sur-Yvette restent stationnaires. Ce n'est qu'au début de septembre que les ruches importées commencent à récolter le pollen de Callune en quantités notables. Enn fin de saison l'écart est considérable : $33 \mathrm{~kg}$ de pollen au total pour les ruches landaises contre $20 \mathrm{~kg}$ pour les ruches de Buressur-Yvette, l'essentiel de la différence étant d'ailleurs représenté par du pollen de Callune. Les figures $\mathrm{I}_{3}$ et $\mathrm{I} 4$ qui font disparaître les différences quantitatives montrent bien que le rythme de la récolte du pollen est différent selon l'origine géographique des colonies. On rapprochera utilement les figures I3 et I4 des figures 3 et 4 . 


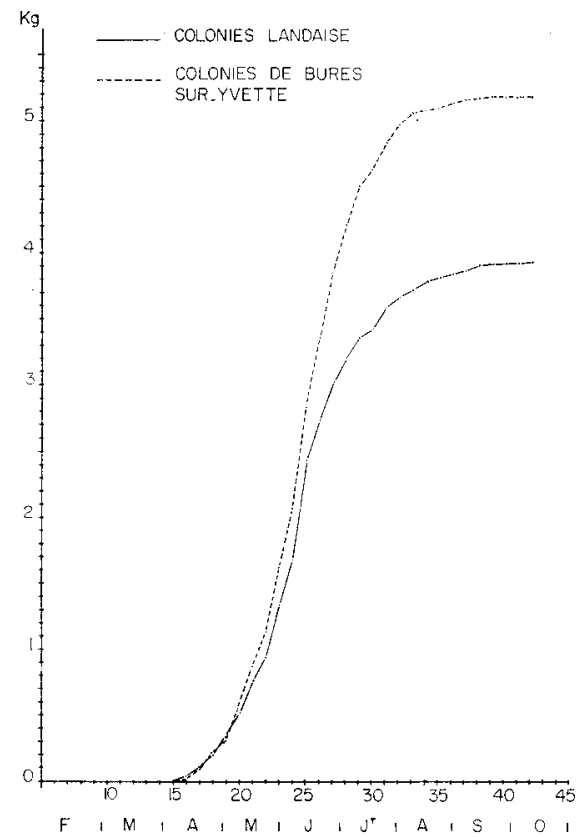

FIG. I2. - Courbes cumulatives des récoltes de pollen à Bures-sur-Yvelte Année I964

D'une part, moyenne des récoltes des colonies originaires de Bures-sur-Yvette. D'autre part, moyenne des récoltes des colonies landaises. Poids exprimés en valeur absolue.

TABLEAU I

Récolte du pollen

(année I963)

\begin{tabular}{|c|c|c|c|c|c|c|}
\hline & \multicolumn{6}{|c|}{ Ruches } \\
\hline & \multicolumn{3}{|c|}{ Transhumantes } & \multicolumn{3}{|c|}{ Sédentaires } \\
\hline & No 1 & No 2 & No 3 & No 35 & $N^{\circ} 40$ & No 44 \\
\hline $\begin{array}{l}\text { Période du } 14 \text { mars } \\
\text { au } 3 \text { juillet } 1963:\end{array}$ & & & & & & \\
\hline Poids en grammes .. & 2,761 & 2,155 & 1,465 & 1,960 & 886 & 1,291 \\
\hline P. 100 du total .... & 94,5 & 74 & 73 & 68 & 67 & $6 x$ \\
\hline $\begin{array}{l}\text { Période du } 3 \text { juillet à } \\
\text { octobre 1963: }\end{array}$ & & & & & & \\
\hline Poids en grammes ... & 154 & 743 & 534 & 491 & 426 & 729 \\
\hline P. 100 du total $\ldots$ & $\begin{array}{c}5,5 \\
\text { (orpheline) }\end{array}$ & 26 & 27 & 32 & 33 & 36 \\
\hline $\begin{array}{l}\text { Poids total du pollen } \\
\text { récolté } \ldots \ldots \ldots \ldots\end{array}$ & 2,915 & 2,898 & 1,999 & 1,557 & 1,312 & 2,020 \\
\hline
\end{tabular}

Les ruches transhumantes sont restées en Chalosse jusqu'au 3 juillet avant de venir dans la Grande-Lande. Les ruches sédentaires sont restées toute l'année dans la Grande-Lande. 
Poids des ruches. - La variation du poids des ruches se fait selon un processus très comparable à la variation du poids des récoltes de pollen. Les courbes de poids sont parallèles à Bures-sur-Yvette et à Sabres pendant tout le printemps. (fig. I5, I6 et I7). A Bures-sur-Yvette ce sont les ruches indigènes qui prennent le plus de

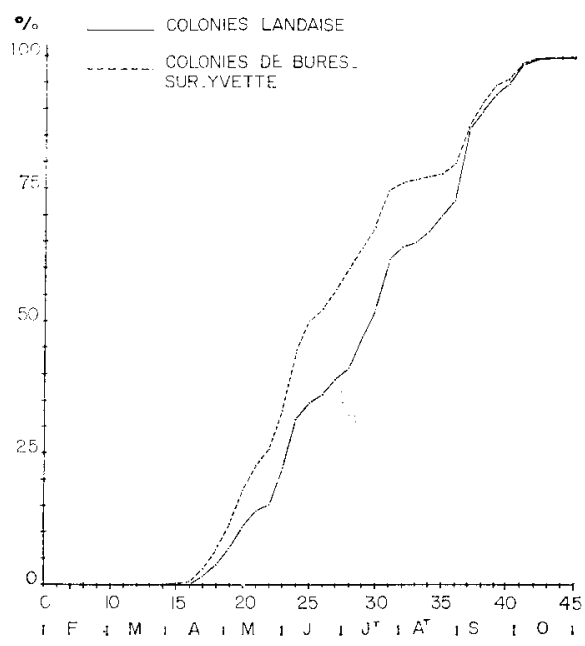

FIG. 13. - Données identiques à celles de la fig. 11 converties en p. 100 de la récolle totale

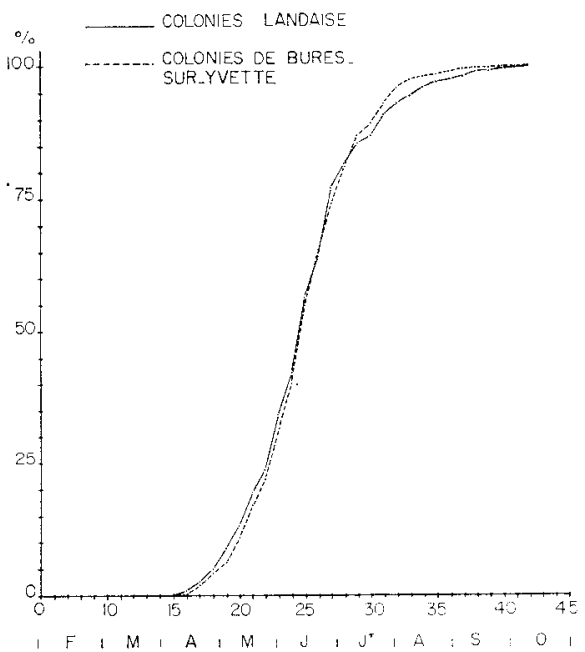

FtG. I4. - Données identiques à celles de la fig. 12 converties en p. 100 de la récolte totale

poids en fin de printemps et début d'été. La différence atteignant en moyenne $6 \mathrm{~kg}$ par colonie au $I_{5}$ juillet. Les courbes sont ensuite parallèles jusqu'en octobre. A Sabres, le parallélisme des courbes est rigoureux jusqu'à la mi-juillet; à partir de ce moment les ruches indigènes perdent plus de poids que les ruches importées, ce qui est dù à un élevage plus intense, mais dès les premiers jours de la floraison de 


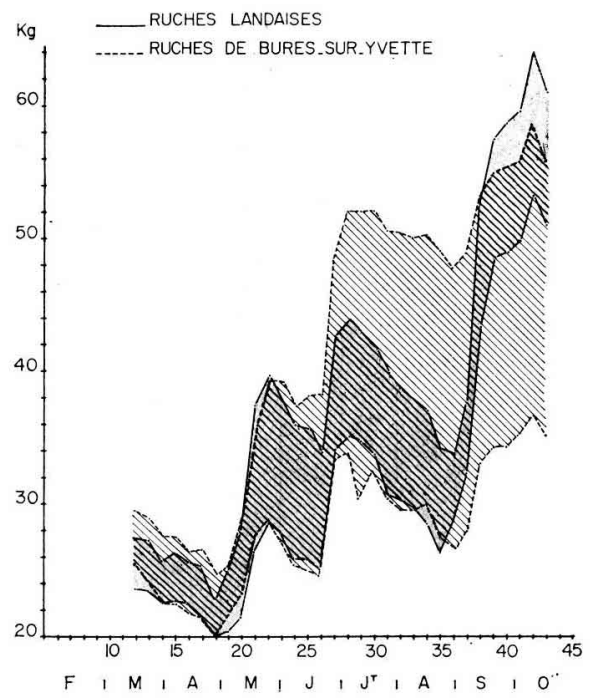

FIG. I5. - Variations de poids des ruches à Sabres.

Année 1964

D'une part espace de variation du poids des ruches landaises

D'autre part espace de variation du poids des ruches originaires de Bures-sur-Yvitte

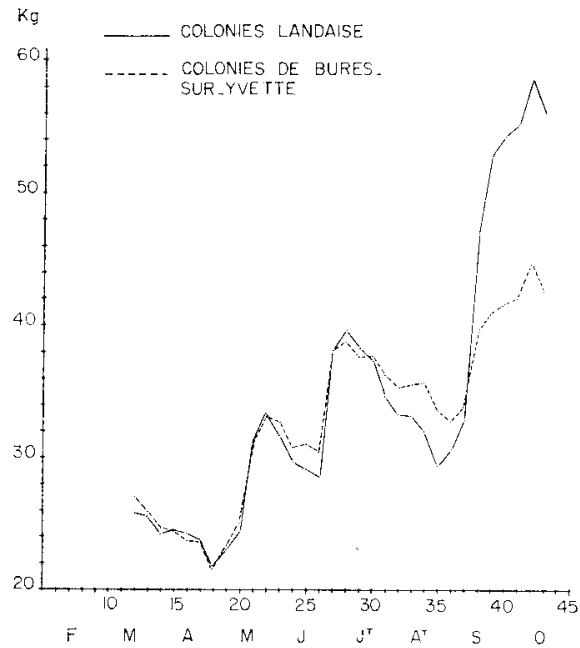

F1G. 16. - Variation de poids des ruches a Sabres. Année 1964

D'une part, moyenne de poids des ruches landaises D'autre part, moyenne de poids des ruches originaires de Bures-sur-Yvette 
la Callune les ruches landaises présentent des gains de poids beaucoup plus importants et finissent la saison avec un excédent de I4 $\mathrm{kg}$ par colonie par rapport aux ruches importées de Bures-sur-Yvette.

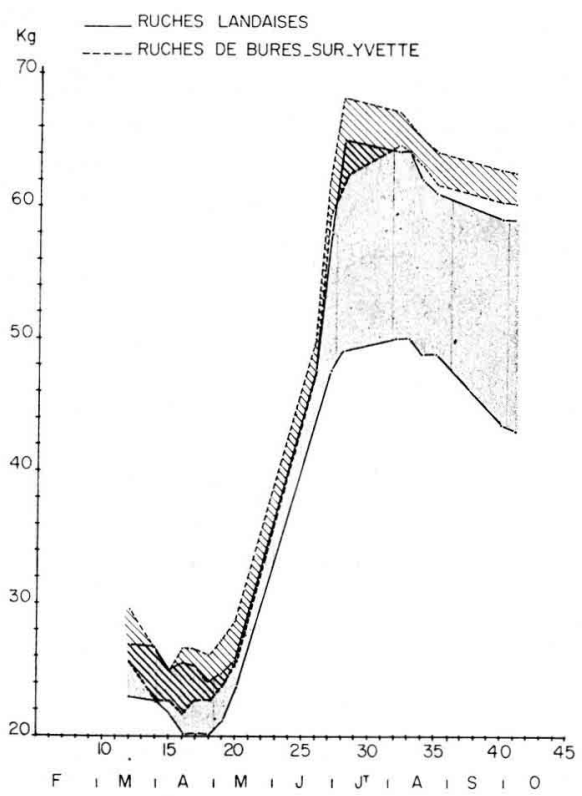

Fic. I7. - Variations de poids des ruches à Bures-sur-Yvetle.

Année 1964

D'une part, espace de varistion du poids des ruches originaires de Bures-sur-Yvette

D'autre part, espace cle variation du poids des ruches landaises

On a éliminé les ruches 17 et 382 (essaimage-orphelinage). Il reste 4 ruches dans chaque lot

Surfaces de couvain. - Nous retrouvons avec les surfaces de couvain un phénomène parallèle aux deux premiers. Les courbes obtenues sont très comparables jusqu'en mai et divergent ensuite plus ou moins. A Bures-sur-Yvette on note une tendance des ruches landaises à poursuivre plus longtemps l'élevage que les ruches indigènes. A la fin du mois d'août, les colonies importées ont 42 points de couvain contre 25 pour les colonies indigènes en moyenne. A Sabres les différences sont beaucoup plus importantes; à la même époque les colonies importées n'ont que 28 points de couvain contre roo pour les ruches indigènes, ce qui montre bien que l'élevage est très fortement réduit dans les ruches importées ; dans les ruches indigènes il a le même niveau qu'en mai, ce qui est l'indice d'une grande activité (fig. I8 et I9).

Il semble qu'on puisse interpréter comme suit les résultats obtenus en I964, année qu'on peut considérer comme normale et même bonne aussi bien à Buressur-Yvette qu'à Sabres. Jusqu'au I5 mai toutes les colonies d'un même lieu d'expérience se comportent d'une façon sensiblement identique; sans doute les facteurs du milieti qui agissent sur les colonies pendant cette période sont-ils insuffisamment discriminants. Aussi bien sur le plan du poids des ruches que sur celui des récoltes de pollen ou de l'étendue du couvain il est impossible de mettre en évidence des différences systématiques. Il n'en est plus de même à partir du I5 mai : 
A Bures-sur-Yvette - A cette date commence la période des grandes récoltes de pollen et de nectar; les ruches indigènes exploitent au maximum les ressources disponibles, ce que ne font pas les abeilles importées des Iandes qui prennent un retard important. A partir d'août, les abeilles indigènes sont pratiquement en hivernage : perte régulière de poids, arrèt de la ponte. Les abeilles landaises ne sont pas dans les mêmes dispositions : leur élevage n'a pas cessé et elles continuent à récolter le peu de pollen disponible. Il semble qu'elles soient à ce moment à la recherche d'une floraison que le milieu ne peut pas leur offrir puisque la Callune n'y existe pratiquement pas et que toutes les floraisons importantes sont passées.

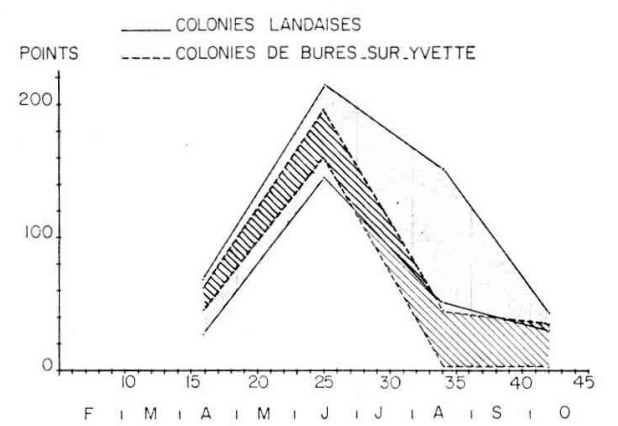

Fig. I8. - Courbes de surjace du convain à Sabres Année 1964

D'une part, espace de variation des surfaces des colonies landaises D'autre part, espace de variation des surfaces des colonies originaires de Bures-sur-Yvette Sauf 19 et 502 (Essaimage - orphelinage). Il reste 4 ruches dans chaque lot

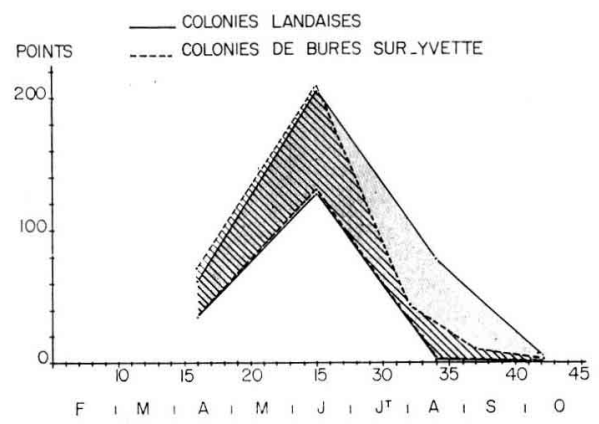

FIG. I9. - Courbes de surface du couvain à Bures-sus-Yvelle Année 1964

D'une part, espace de variation des surfaces des colonies originaires de Bures-sur-Yvette. D'autre part, espace de variation des surfaces des colonies landaises

On a éliminé les ruches 382 et 17 (Essaimage - orphelinage). Il reste 4 ruches dans chaque lot.

A Sabres - Toujours à partir du $\mathrm{I}_{5}$ mai, les ruches indigènes prennent un léger avantage sur les ruches importées de Bures-sur-Yvette en ce qui concerne la récolte du pollen ; elles semblent mieux adaptées à l'utilisation d'une flore pauvre ; progressivement elles se développent, quitte à perdre du poids en juillet et même en août pour brusquement, lorsque fleurit la Callune, dépasser brutalement les ruches importées qui, s'étant mises plus ou moins en hivernage n'ont pas pu exploiter une miellée aussi tardive. 
Ce qu'il faut encore noter (fig. I5 et I7) c'est que les courbes de variation de poids des ruches montrent dans les deux cas une forte concentration pour les ruches indigènes et une forte dispersion pour les ruches importées comme si la transplantation déclenchait des réactions au hasard alors que le travail de l'Abeille indigène se ferait selon un programme précis.

Les résultats obtenus en I964 étaient encourageants en ce sens qu'ils démontraient assez clairement l'existence d'un phénomène d'adaptation de l'Abeille d'une localité au régime local du climat et de la flore. Il n'en reste pas moins vrai que nous n'étions pas plus avancés en ce qui concerne l'explication du phénomène. Ne pouvant exploiter à la fois toutes les idées qui se présentaient nous avons tenté de rechercher quel pouvait être le rôle de la reine dans ce phénomène d'adaptation.

\section{Résultats d'une expérience de permutation de reines}

Au cours de 1'année $\mathrm{r} 965$ nous avons poursuivi l'exécution de notre programme et mis en place l'expérience suivante. Le $\mathbf{z} 7$ juin, dix colonies d'abeilles landaises qui avaient été orphelinées quelques jours auparavant furent remérées avec de jeunes reines fécondes obtenues à partir de deux élevages différents. Cinq colonies prises au hasard reçurent une reine élevée et fécondée à Sabres à partir d'une souche landaise; cinq autres colonies reçurent une reine expédiée de Bures-sur-Yvette et provenant d'un élevage fait à la station sur une souche de la région parisienne. A partir de l'introduction des jeunes reines on procéda aux observations habituelles.

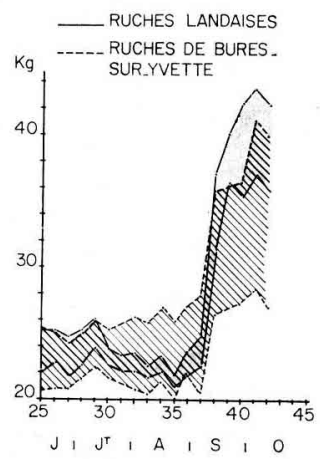

FIG. 20. - Variations de poids des ruches à Sabres

Année 1965

D'une part, espace de variation du poids des ruches landaises (sauf $n^{\circ}$ I 5 orpheline).

D'autre part, espace de variation du poids des ruches avec reines originaires de Bures-sur-Yvette.

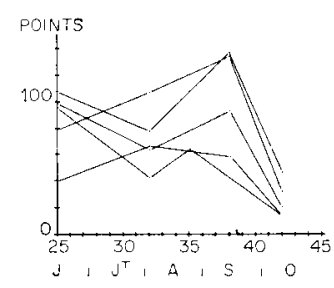

FIG. 2I. - Courbes de surface du couvain à Sabres

Année I965

Colonies avec reine originaire de Bures-sur-Yvette.

Les figures 20 à 23 traduisent les résultats obtenus. Les colonies dotées d'une reine landaise ont eu un comportement qu'on peut considérer comme assez homogène, surtout en ce qui concerne les variations de poids (fig. 2o) ; les courbes sont très groupées alors que celles qui correspondent aux colonies dotées d'une reine élevée à Bures-sur-Yvette sont très dispersées ; les meilleures récoltes sont le fait des colonies purement landaises. La comparaison des courbes de développement du couvain 
montre un phénomène identique. Toutes les reines landaises n'ont pas la même valeur comme pondeuses mais toutes fournissent des courbes de surface du couvain dont l'allure générale est la même : partie ascendante jusqu'en août, palier jusqu'au I5 septembre. Avec les reines importées les discordances sont grandes (fig. 2I) ; trois courbes sur cinq sont inversées par rapport aux courbes fournies par les teines landaises. La récolte du pollen est plus importante pour les colonies dotées d'une reine landaise mais la dispersion est grande dans les deux lots; on notera toutefois que si la quantité de pollen récoltée varie du simple au double pour les colonies ayant reçu une reine landaise elle varie du simple au triple pour les colonies ayant reçu une reine importée (fig. 23).

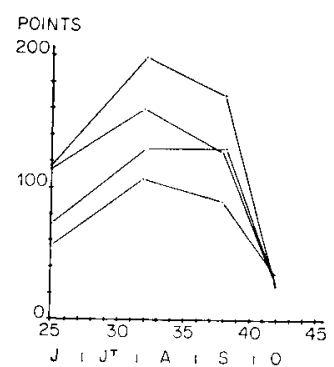

FIG. 22. - Courbes de surface da courain à Sabres Année 1965

Colonies avec reine Landaise (sauf $n^{0} 15$ orpheline)

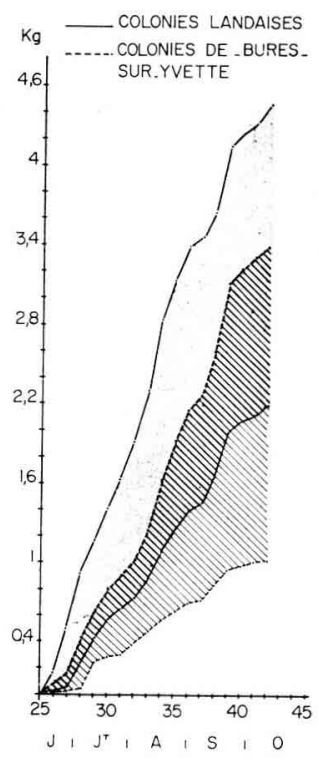

Fis. 23. - Courbes cumulatives de la récolle di pollen à Sabres

Année 1965

$D^{\prime}$ une part, espace de variation des colonies lanlaises (sauf $n^{0}$ i 5 orpheline).

D'autre part, espace de variation des colonies avec reine de Bures-sur-Y vette.

Les expériences de 1965 demanderaient à être reprises sur une plus grande échelle et dans des conditions plus rigoureuses en ce qui concerne la fécondation des reines utilisées. Fin effet, la descendance des reines fécondées à Bures montra par la suite dans certains cas des signes d'hybridation partielle avec de l'Abeille italienne; ceci n'empêche pas, bien entendu que les reines elles-mêmes étaient de pure race noire et, dans la mesure oì l'on s'intéresse au rôle de la reine, la façon dont elle a été fécondée peut être considérée comme secondaire.

Si 1'on examine ce qui s'est passé dans les dix ruches en expérience à partir du moment où la nouvelle reine a été introduite, on constate que jusqu'au Io juillet environ elles ont vécu avec une population adulte exclusivement landaise ; les premières butineuses issues de la nouvelle reine ne sont apparues qu'au début du mois 
d'août; les filles de la reine précédente n'ont été totalement éliminées que vers la fin du mois d'août. Étant donné qu'on constate dès le $\mathrm{I}^{\mathrm{er}}$ août des différences très importantes entre les deux lots, à tous les points de vue, il faut bien admettre que la reine a joué un rôle essentiel dans les événements. Il serait sans doute prématuré de conclure que les ouvrières ne jouent aucun rôle et que la reine détient seul les clefs de 1'adaptation de la colonie à son milieu naturel; d'autres expériences devront encore être faites pour aboutir à des conclusions plus solides.

\section{DISCUSSION}

Les observations et les expériences qui viennent d'être exposées appellent un certain nombre de remarques théoriques et pratiques.

Il semble bien établi que le cosmopolitisme de 1'abeille soit en rapport avec ses grandes facultés d'adaptation aux différents types de climat et de flore. On peut penser avec Avertisyan (I959) que les Apides, ayant évolué parallèlement aux Phanérogames entomophiles dont ils tirent leur alimentation et dont ils assurent la pollinisation, ont conquis en même temps que ceux-ci les différents milieux naturels qui s'offraient à leur extension. Au sein du genre $A$ pis les espèces les plus primitives (Apis florea, Apis dorsata) seraient liées aux conditions d'existence les plus faciles, c'est-à-dire celles des régions chaudes à flore exubérante. La conquête des milieux présentant des conditions de vie difficiles ou très difficiles (hiver longs et rigoureux, flore pauvre) n'aurait été possible que par les espèces et races les plus évoluées présentant les caractères adaptatifs indispensables : colonies populeuses, activité de butinage très développée, résistance au froid, langue plus longue, etc. AvETISYAN voit dans ce mode d'évolution l'origine des qualités indéniables de robustesse et d'activité des abeilles montagnardes du Caucase. En conclusion, les différentes espèces et races d'abeilles existant dans le monde représenteraient des types évolutifs adaptés à des conditions écologiques déterminées. Par voie de conséquence, en chaque lieu, la race locale d'Abeille serait toujours par définition, la mieux adaptée. Braun (I955) exprime la même idée.

Pour vérifier la valeur de l'hypothèse d'Avetrsyan il convient de répondre à deux questions. La première est de savoir si, en un lieu donné, il existe bien un. régime bioclimatique suffisamment stable pour constituer la base d'une éventuelle adaptation. La seconde concerne l'existence dans la colonie d'abeilles d'un cycle évolutif annuel prédéterminé. De la réalité des deux phénomènes dépend, bien entendu, la possibilité d'une action du premier sur le second.

La stabilité du régime bioclimatique paraît bien établie. Les observations dont nous disposons et qui ont été exposées plus haut montrent qu'à Bures-sur-Yvette comme à Sabres, chaque année ramène avec régularité un cycle de floraisons déterminé. Par ailleurs, les recherches de Koch (voir plus haut) relatives à ce même problème sont très convaincantes. Les très nombreuses observations effectuées pendant des dizaines d'années dans la R. D. A. ont montré qu'on peut distinguer dans ce pays, en fonction de la nature du sol, de la flore et du climat, des zones caractérisées par tun régime particulier. KocH a pu reconnaître cinq régimes qui sont : vernal précoce, vernal tardif, vernal contrarié, forestier et estival. Chacun de ces régimes 
est caractérisé par un type de courbe de variation du poids des ruches. Les types de courbes présentent une grande régularité, c'est-à-dire qu'en un lieu donné on retrouve chaque année les mêmes pics sinon aux mêmes dates précises du moins aux mêmes périodes. Les pics correspondent eux-mêmes à des floraisons essentielles et ils ne sont jamais très nombreux au cours d'une année. Les données recueillies par $\mathrm{KOCH}$ sont suffisamment nombreuses pour que cet auteur ait pu établir une carte très détaillée des régimes bioclimatiques apicoles de 1a R. D. A.

La réponse à la première question est donc affirmative. La notion de régime bioclimatique local constitue une donnée solide. Elle trouve également un appui certain dans les travaux de JEFFREE (I957) qui ne sont pas sans rapport avec ceux de Косн bien qu'ils se placent sur un plan différent.

Du côté de l'abeille, si nous nous reférons à la masse des observations faites tant par les biologistes que par les praticiens, il paraît indéniable que chaque race géographique d'abeilles possède son rythme propre de développement. Si l'on considère comme point de comparaison, d'une part les dates du début de la ponte, d'autre part le nombre des individus adultes ou larvaires présents à chaque moment de l'année, on constate que les variations sont grandes d'une race à l'autre pour un même lieu. On constate aussi des différences dans le comportement de butinage (Louveaux, I958; Bornus et al., I96r ; Warakomska et Louveaux, 1964).

Étant donné qu'il existe bien un régime bioclimatique local et un cycle évolutif annuel prédéterminé chez l'Abeille, toute opération de déplacement d'abeilles d'une race géographique donnée vers un lieu où elle est étrangère doit avoir des conséquences mesurables. Il en est bien ainsi selon les différents auteurs ayant étudié cette question: Lunder, I953; FotT, I956 ; BilaAS, I958; RAGIM-ZADE, I963; Mirza, I964; MeI Nitchenko, I965.

Tous ces auteurs ont mis en évidence des différences de comportement et une tendance à la conservation du type de cycle évolutif annuel caractéristique de la race. Cette tendance peut d'ailleurs se manifester aussi bien à l'avantage qu'au détriment des colonies déplacées.

Les résultats que nous avons obtenus en travaillant à l'intérieur d'une même race géographique et qui ont été exposés plus haut montrent que ce que l'on savait des races géographiques s'applique aussi aux souches locales. Le passage d'un régime vernal précoce à un régime estival et vice versa, se traduit chez l'Abeille dépaysée par un comportement mal adapté. Les expériences de 1965 portent à croire que la reine est dépositaire du "programme " selon lequel la colonie évolue au cours de 1'année; dès son introduction dans une colonie étrangère elle semble bien imposer, à travers son rythme de ponte, la réalisation des différentes étapes du cycle biologique. Son rôle ne serait pas seulement celui qu'on lui attribue souvent de " machine à pondre "mais aussi celui de régulateur.

Tout semble donc se passer comme si les colonies d'abeilles d'un lieu donné avaient connaissance d'un certain nombre de données bioclimatologiques relatives à ce lieu (longueur des jours, température moyenne, rythme des floraisons) et adaptaient leur cycle biologique annuel à ces données dont l'ensemble constitue un régime. Étant donné que l'essentiel pour la colonie est de pouvoir essaimer puis de constituer ses réserves hivernales, on conçoit toute l'importance pour les abeilles livrées à leurs seules ressources d'ajuster leur comportement aux conditions locales ; toute 
activité à contre temps se retourne contre la colonie et peut entraîner sa disparition. On peut donc envisager raisonnablement que, dans une certaine mesure, la qualité de l'adaptation au milieu naturel soit un facteur de sélection assez draconien.

Le problème de la nature profonde de l'adaptation constatée (caractère génétique ou accomodat) reste à peu près entier, même si l'on tient compte des observations qui permettent de penser que la reine joue un rôle essentiel dans le phénomène. Nous ne savons pas si l'adaptation à un régime bioclimatique donné est ou n'est pas labile. Une colonie adaptée à un régime estival peut-elle progressivement s'adapter à un régime vernal précoce, par exemple? Nous n'en savons rien pour le moment. Il est possible, mais non certain, que les acclimatations constatées par les praticiens soient le fait d'un renouvellement de la reine et qu'il soit dû, par conséquent, au croisement avec les souches locales voisines. On sait simplement que dans le cas de races géographiques vraies il n'y a pas adaptation totale tant que la souche reste pure. Ce genre de problème ne pourra être approfondi que par une étude génétique appropriée portant sur 1'héritabilité des caractères adaptatifs. De nouvelles expériences sont nécessaires, notamment avec l'aide de l'insémination artificielle.

Sur le plan pratique, il semble qu'on puisse faire dès maintenant un certain nombre de remarques sur le travail réalisé. Il faut tout d'abord se garder de conclure que l'Abeille locale étant bien adaptée au régime bioclimatique du lieu où elle vit est forcément la meilleure pour l'apiculteur. En effet, l'adaptation est en rapport avec la nécessité pour l'Abeille de boucler son cycle annuel dans les meilleures conditions et non avec les éventuels profits de 1'apiculteur. Il est probable que si l'Abeille landaise est médiocre au printemps du point de vue apicole c'est que le développement précoce des colonies dans le biotope landais constituerait une erreur biologique aboutissant au gaspillage des réserves. De même l'Abeille de régime vernal précoce n'a aucun besoin réel des miellées tardives et ne s'y intéresse pas (en tout cas ne les prévoit pas dans son " programme »).

Ces considérations sont de nature à remettre en cause les bases mêmes de la sélection des abeilles. Une sélection valable en régime estival ne l'est pas forcément en régime vernal. Les appréciations très diverses formulées à propos des différentes races et souches disponibles actuellement ne sont sans doute pas sans rapport avec le fait qu'une abeille ne saurait convenir à tous les biotopes. On peut aussi se demander si l'hybridation n'a pas pour conséquence de faire disparaître l'adaptation à un régime bioclimatique déterminé ; il y aurait alors libération totale de l'instinct reproducteur et disparition de tout ce qui constitue le frein biologique indispensable. On sait que beaucoup d'abeilles très hybridées ou fortement sélectionnées pour la ponte semblent avoir perdu toute notion de l'importance des réserves de nourriture; la reine ne ralentit pratiquement pas son activité génésique et la ruche ne peut survivre dans beaucoup de régions que si l'apiculteur lui fournit le sirop de sucre à volonté. De telles colonies n'ont un intérêt que dans des régions de miellée continue et sont sans valeur pour tirer profit de miellées courtes et peu intenses. D'un autre côté il est vraisemblable que les races et souches très pures présentent au maximum des caractères adaptatifs qui en limitent 1'utilisation. L'abeille des bruyères du nord de l'Allemagne pen utilisable en dehors des régions de landes est probablement un bon exemple dans ce sens. De même pour notre abeille landaise.

Le fait que l'agriculture évolue rapidement entraîne certainement des modifications profondes dans le régime bioclimatique de certaines régions; les cultures 
changent et la destruction systématique des mauvaises herbes ainsi que le déchaumage et l'abattage des haies constituent des facteurs très importants de modification du biotope. D'autre part, la pratique de l'apiculture pastorale se généralise. Dans ces conditions, on peut se demander comment réagit l'Abeille et si les mauvais résultats enregistrés dans certaines régions ou pour certains types de transhumance n'ont pas pour origine un manque d'adaptation des abeilles à des fluctuations de régime continuelles.

Faisant la part de 1'hypothèse qui reste encore très importante dans ces conclusions, il semble cependant qu'il soit prudent, dès maintenant, de reconsidérer les problèmes de sélection et d'envisager deux voies distinctes : sélection de souches hautement adaptées à un régime bien précis (abeiles destinées à la miellée de Callune ou à celle du miellat de Sapin par exemple) et production d'hybrides au comportement plastique pouvant donc s'adapter aux circonstances les plus diverses sans toutefois devenir ces monstres de fécondité que nous connaissons et dont le mécanisme prévisionnel est totalement déréglé.

I)'autres questions restent pendantes. Quel genre de relation existe-t-il entre le régime bioclimatique et la nutrition de la colonie, ainsi que son état sanitaire? Nous n'en savons pas grand-chose sinon que tous les pollens n'ont pas la même valeur biologique (Maurizio, I954) ni la même composition chimique. Peut-être, le cycle annuel des colonies est-il en partie sous la dépendance de substances présentes dans les pollens et nécessaires à la fécondité. Il n'est peut-être pas indifférent qu'une colonie s'alimente sur Rosacées et Papilionacées ou sur Éricacées. Il y a là encore un vaste domaine à explorer. De même, nous ne savons pas grand-chose des chimiotropismes qui dirigent l'abeille vers les différentes sources de pollen. Il serait intéressant de comparer le cycle de l'alimentation des colonies dans divers régimes bioclimatiques en reprenant les observations faites antérieurement, notamment sur la variation saisonnière de la richesse en azote de la récolte de pollen (LOUVEAUX, I958).

Nous avons conscience d'avoir abordé un sujet qui, vu de l'extérieur parait mieux connu des praticiens que des biologistes. Malheureusement l'expérience des praticiens reste le plus souvent inédite et il est très difficile de l'exploiter de façon rationnelle. Souhaitons donc que les apiculteurs de métier sortent de leur réserve et nous fassent savoir dans quelle mesure leur expérience personnelle concorde avec nos observations. Ils pourront ainsi nous aider à préciser l'orientation des recherches futures dans un domaine qui apparaît dès maintenant comme d'une grande importance.

Reçu pour publicauon en notrembre 1966.

\section{SUMMARY}

ADAPTATIONS OF THE BEE " AI'IS MELLIFICA " L. TO NATLRAL HABITAT

Different geographical breeds of the bee (Apis mellifica I.) have anatomical and physiological characteristics which can be considered to be adaptations to the habitat of their origin. These adaptive characteristics continue to be shown with greater or lesser clarity when colonies of a given breed are transported to a new environment. Little is known however of the adaptive characte- 
ristics shown by local varieties of the bee, which are doubtless only accommodations and cannot be used taxonomically.

Three habitats very different in flora and climate were studied; the Paris basin (in the region of Bures-sur-Yvette), the great heath of Gascony (in the region of Sabres), and Provence (in the region of Montfavet near Avignon). The data collected show that Bures-sur-Yvette belongs to the early vernal bioclimatic system, Sabres is connected with the purely aestival system, while Montfavet represents an early vernal an secondarily aestival type (fig. I to Io). A comparison is made with KoCH's work ( 1962 to Ig66).

When bees originating in Bures-sur-Yvette (early vernal system) were transported to Sabres (aestival system) and vice versa, they proved to be unadapted to the new environment, and this was shown by worse performances (weight of hive, pollen harvest, extent of the brood-comb) than those of the indigenous colonies (fig. II to I9).

It seems then that bees adapt to a certain bioclimatic system, and a change of system perturbs the life of the colony. A change of the queen has the same effect, which leads to the supposition that to a certain extent the queen controls the annual biological cycle of the colony (fig. 20 to 23).

Finally, the importance of the idea of adaptation in the sphere of bee selection is discussed. Too narrow an adaptation to a given habitat could be a disadvantage and could impede diffusion of the selected strain, and yet the loss of adaptation, to a lesser or greater degree, could be an advantage in the sense that the bee would retain a greater plasticity of behaviour.

\section{RÉFÉRENCES BIBLIOGRAPHIQUES}

Avetisyan G. A., I958. On certain problems of evolution, geographical distribution, protection and utilization of species and races of bees. C.R.XVII $\mathrm{e}$ Congr. inlernation. Apicull. Rome, 1958, 2, 223-226

Bilash G. D., I958. La production du miel de différentes races d'abeilles en rapport avec le type de développement des colonies et les particularités de la récolte (en russe). Byull. nauch-lech. Inf. Inst. Pchelovodstza $(3 / 4): 9^{-1} 2$.

Bornus L., FABIANSKi A., Lecewicz W., i96r. La récolte du miel par trois races d'abeilles à Pulawy (en polonais). Pszczel. Zesz. Nauk., 5 (2), 85-94.

Braun E., I955. Klima und Weide schufan ihre Bienen. Dische Bienemwirtschaft, 6 (I0), 213-2r5.

Covturier A., I950. L'apiculture de la région landaise. VIIIe Congr. inlernalion. Entomol., Stockholm, 3948, p. $5^{8-65}$.

Cúnot L., Tétry A., 1951. L'évolution biologique, 592 p. Masson et C'ie, Paris.

Droege G., 1960. Zur Frage der Unterscheidbarkeit der Carnika-Stämme. Arch. Geflügelsucht Kleinlierkde, 9 (3), $182-203$.

Foтг X., 1956. Données préliminaires sur le comportement d'abeilles des steppes, du Banat et de la Transylvanie dans les conditions de la steppe (en roumain). Anal. Inst. Cerc. Zoot., 14, 633-641.

Fresnaye J., ig6r. Deux nouveaux appareils pour la pesée des ruches. Ann. Abeille, 4, 36i-367.

Fresnaye J., 1962. Un appareil pour le calcul rapide des surfaces de couvain dans les ruches. Ann. Abeille, 5, $145^{-\mathrm{I}} 53$.

Fresnaye J., 1963. Les erreurs d'orientation des abeilles (dérive) dans le rucher modeme. Ann. Abeille 6, $185^{-200}$.

FRESNAYE J., 1965. Étude biométrique de quelques caractères morphologiques de l'abeille noire française (Apis mellifica mellifica). Ann. Abeille, 8, $27 \mathrm{I}-283$.

GoETzE G., 1964. Die Honigbiene in natürlicher und kïnstlicher Zuchtauslese. :

Teil I : Systematik, Zeugung und Vererbung, Izo p.

Teil II. : Beurteilung und züchterische Auslese von Bienenvölker, 92 p.

Monographien zur angew. Entomologie. Beih. Z. angew. Entomol., $\mathrm{n}^{\mathrm{so}} \mathrm{I} 9$ et 20. Paul Parey. Hambourg et Berlin.

JEFFREE E. P., I957. Some aspects of the seasonal course of honeybee colonies and the changing background of flowering plants on which they forage, Univ. Aberdeen : D. Sc. thesis, $324 \mathrm{p}$.

Косн H. G., 1962. Die Entwicklung der Bienenvölker und die Nektartracht in ihren Beziehungen zur Witterung. Biometeorology, 531-536.

Косн H. G., 1964. Von Wert der Waagstockbeobachtungen. Garlen und Kleinlierzucht, 3 (1о), 6-7.

Kосн H. G., r $965 a$. Die Regionale Verteilung der Honigtracht im Gesamtgebiet der D. D. R. auf Grund von Waagstockbeobachtungen. $X X^{\mathrm{e}}$ Congr. internation. Apicult., Bucarest.

Косн H. G., $1965 b$. Die Trachtverhältnisse auf norddeutschen Sandböden. Garten und Kleintierzucht., 4 (I2), 8-10.

Косн H. G., r965c. Die Trachtmöglichkeiten in der D. D. R. Garten und Kleintierzucht, 4 (Ir), 8-9. Annales de l'Abeille. - 1966. 
Koci H. G., 1966. Die Trachtgebiete der D. D. R. und ihre räumliche Verteilung. Garten lund Kleintieratucht, 4 (2), 8-9, 14 .

Lavie P., Fresnaye J., I963. Étude expérimentale de la trappe à pollen en position supérieure. Ann. Abeille, 6, 277-30r.

Lous J., тg63. Étude de la translation du point discoïdal (Diskoidalverschiebung) de l'aile de l'Abeille (Apis mellifica). Ann. Abeille, 6, 303-320.

LOUvEayx J., I958. Recherches sur la récolte du pollen par les abeilles (Apis mellifica L.), zos p. I. N. R. A. Paris. These.

Louveavx J., 1965. Die Probleme der Akklimatisierung bei der Honigbiene. Dische Bienenvirtschaft, 16, 313-316.

Louveaux J., Albiserti J., I963. Observations préliminaires sur la récolte du pollen par les abeilles dans les "Grandes Landes" de la forêt landaise. Ann. Abeille, 6, 229-234.

Lunder R., I963. Comparaison de différentes races d'abeilles sous les conditions de la Norvège (en norvégien). Nord. Bilidskr., 5, 7 $1-83$.

MaURizio A., I054. Pollenemährung und Lebensvorgänge bei der Honigbiene (Apis mellifica L.). Landw. Jahrbuch Schweiz., 68, Ir 5-18.

Melnitcienko A. N., I965. Les modifications des aptitudes vitales et productives des abeilles de différentes races déplacées dans des zones bioclimatiques nouvelles (en russe). Vedecke Prace, 4, I I 7-124.

Mirza E., I964. Données comparatives sur le comportement des abeilles dans les régions de steppes et dans les montagnes (en roumain). Apicultura Bucuresti, 17 (6), I-8.

RAGIM-ZADE M. C., I 963 . The effect of climatic conditions upon the efficiency of different races of Apis mellitera L. (en russe). Rev. Entomol. U. R. S. S., 4, 770-777.

Rutroner F., ig63. Die Zuchtauslese bei der Biene. 79 p. N. O. Imkerschule Wr. Neustadt.

Warakomska Z., Louveaux J., Ig64. Recherches sur les variations du comportement de butinage chez. des colonies d'abeilles de races différentes. Ann. Abeille, 7, 217-31. 